\title{
reciamuc
}

Revista cientifica de investigación actualización del mundo de las ciencias

\author{
Diana Stefania Evangelista Barragan ${ }^{\text {a }}$; Gabriela Estefania Jalon Flores ${ }^{\mathrm{b}}$; Jorge \\ Efraín Camacho Desidero ${ }^{c}$; Johamelly Maritza Peña Martillo ${ }^{d}$
}

Dermatitis atópica (ECCEMA) infantil

Child atopic dermatitis (ECCEMA)

Revista Científica de Investigación actualización del mundo de las Ciencias. Vol. 3 núm., 3, julio, ISSN: 2588-0748, 2019, pp. 192-207

DOI: 10.26820/reciamuc/3.(3).julio.2019.192-207

URL: $\underline{\text { http://reciamuc.com/index.php/RECIAMUC/article/view/274 }}$

Código UNESCO: 3205 Medicina Interna

Tipo de Investigación: Artículo de Revisión

(C) RECIAMUC; Editorial Saberes del Conocimiento, 2019

Recibido: 28/04/2019

Aceptado: 19/05/2019

Publicado: 01/07/2019

Correspondencia: dianita_evangelista@hotmail.com

a. Master Universitario en Neuropsicología y Educación; Medico; Investigador Independiente; Guayaquil, Ecuador; dianita_evangelista@ hotmail.com

b. Médico; Investigador Independiente; Guayaquil, Ecuador; gabysjalon@ gmail.com

c. Médico; Investigador Independiente; Guayaquil, Ecuador.

d. Médico; Investigador Independiente; Guayaquil, Ecuador. 


\section{Dermatitis atópica (ECCEMA) infantil}

Vol. 3, núm. 3., (2019)

Diana Stefania Evangelista Barragan; Gabriela Estefania Jalon Flores; Jorge Efraín Camacho Desidero; Johamelly Maritza Peña Martillo

\section{RESUMEN}

La dermatitis atópica es uno de los tipos más comunes de eccema, siendo este último término el usado para referirse a varios tipos distintos de hinchazón de la piel, mismos que en su mayoría, causan sequedad, comezón, sarpullido en la cara, al interior de los codos y detrás de las rodillas, en las manos y/o en los pies, no obstante, esta condición no es contagiosa. Su prevalencia en niños pequeños se ha reportado en cifras inclusive superiores al $20 \%$ en países como Estados Unidos, y su incidencia va en aumento en todo el mundo, afectando indistintamente de la raza y la geografía. Cerca del $65 \%$ de los pacientes desarrollan síntomas antes cumplir un año de edad y cerca del $90 \%$ de ellos desarrollan síntomas antes de los 5 años. Algunos niños superan el eczema cuando llegan a ser adultos jóvenes, aunque su piel continúa siendo seca y sensible. El desarrollo de este artículo se llevó a cabo en el marco de una investigación documental, limitado a la búsqueda de material bibliográfico por medio del uso de diversas bases de datos, tales como: MedlinePlus, PubMed, Portal Regional de la BVS, SciELO y ELSEVIER, de las que se fueron seleccionando los materiales bibliográficos que fundamentalmente guardaron mayor relevancia, y además respondieran a los criterios de vigencia y transcripción en los idiomas español e inglés. El objetivo se centra en exponer de manera resumida las conceptualizaciones elementales que la literatura científica más reciente que se encuentra disponible respecto a la dermatitis atópica o eccema atópico en niños, y para ello, se hizo referencia a las definiciones de esta patología, sus causas, características, síntomas, comportamiento infantil, tratamiento farmacológico y cuidados preventivos en el hogar. Se concluye destacando la importancia de las acciones de prevención por parte de adulto responsable del niño teniendo como perspectiva el persistente incremento de su prevalencia, independientemente de que se sigan desarrollando alternativas medicamentosas para mejorar los síntomas y la prevención de esta particular enfermedad.

Palabras Claves: Atópica; Piel; Sensible; Niños; Prevención. 


\title{
Dermatitis atópica (ECCEMA) infantil
}

Vol. 3, núm. 3., (2019)

Diana Stefania Evangelista Barragan; Gabriela Estefania Jalon Flores; Jorge Efraín Camacho Desidero; Johamelly Maritza Peña Martillo

\begin{abstract}
Atopic dermatitis is one of the most common types of eczema, the latter being the term used to refer to several different types of skin swelling, which in most cases cause dryness, itching, rash on the face, inside of the elbows and behind the knees, in the hands and / or feet, however, this condition is not contagious. Its prevalence in young children has been reported in figures even higher than $20 \%$ in countries such as the United States, and its incidence is increasing worldwide, affecting race and geography indistinctly. About $65 \%$ of patients develop symptoms before reaching one year of age and about $90 \%$ of them develop symptoms before age 5 . Some children outgrow eczema when they become young adults, although their skin remains dry and sensitive. The development of this article was carried out in the framework of a documentary research, limited to the search of bibliographic material through the use of diverse databases, such as: MedlinePlus, PubMed, Regional Portal of the VHL, SciELO and ELSEVIER, of those that were selected bibliographic materials that fundamentally kept greater relevance, and also respond to the criteria of validity and transcription in the Spanish and English languages. The objective is to expose in a summarized way the elementary conceptualizations that the most recent scientific literature available regarding atopic dermatitis or atopic eczema in children, and for this, reference was made to the definitions of this pathology, its causes, characteristics, symptoms, child behavior, pharmacological treatment and preventive care at home. We conclude by emphasizing the importance of prevention actions by the adult responsible for the child, taking into account the persistent increase in its prevalence, regardless of whether drug alternatives are still being developed to improve symptoms and the prevention of this particular disease.
\end{abstract}

Key Words: Atopic; Skin; Sensitive; Children; Prevention. 


\section{Dermatitis atópica (ECCEMA) infantil}

Vol. 3, núm. 3., (2019)

Diana Stefania Evangelista Barragan; Gabriela Estefania Jalon Flores; Jorge Efraín Camacho

Desidero; Johamelly Maritza Peña Martillo

\section{Introducción.}

De acuerdo con el Instituto Nacional de Artritis y Enfermedades Musculoesqueléticas y de la Piel de los Estados Unidos de América (National Institute of Arthritis and Musculoskeletal and Skin Diseases / NIAMS, por sus siglas en inglés), la dermatitis atópica es uno de los tipos más comunes de eccema, siendo éste último término el usado para referirse a varios tipos distintos de hinchazón de la piel, mismos que en su mayoría, causan sequedad, comezón, sarpullido en la cara, al interior de los codos y detrás de las rodillas, en las manos y/o en los pies. Esta afección no es contagiosa y su origen es desconocido, no obstante, es probable que su causa se deba a factores genéticos y ambientales. Ocurre con mayor frecuencia en los bebés y niños, sin embargo, también puede afectar a los adultos, pudiendo en cualquiera de los casos desarrollarse fiebre del heno y asma. Es una enfermedad de larga duración, pero en los niños, a medida que crecen, el problema puede mejorar o desaparecer. Es frecuente que a veces la piel pueda permanecer seca e irritarse fácilmente. (NIAMS, 2018)

La prevalencia es de alrededor del $15 \%$ al $20 \%$ en niños y jóvenes [...] En niños pequeños se han reportado cifras de hasta el $20 \%$ en países como Estados Unidos y su incidencia va en aumento en todo el mundo. Afecta a todas las razas y existe en todos los países. Los datos más valiosos sobre dermatitis atópica provienen de International Study of Asthma and Allergies in Childhood (ISAAC), un estudio global sobre alergias con criterios uniformes que abarca unos 2 millones de niños en unos 100 países. Si bien define la prevalencia en más de $20 \%$ de los niños, esta varía significativamente alrededor del mundo. Para el grupo de 6 a 7 años, los datos varían de $0,9 \%$ en India hasta $22,5 \%$ en Ecuador [...] Datos recientes de este estudio muestran que en algunos países se alcanzó un tope de prevalencia como en el Reino Unido y 


\section{Dermatitis atópica (ECCEMA) infantil}

Vol. 3, núm. 3., (2019)

Diana Stefania Evangelista Barragan; Gabriela Estefania Jalon Flores; Jorge Efraín Camacho Desidero; Johamelly Maritza Peña Martillo

Nueva Zelanda, disminuyendo desde ese nivel. Sin embargo, sigue en aumento sobre todo en niños en países de bajos ingresos de Latinoamérica y el sureste de Asia. (NIAMS \& AAD, 2018)

El objetivo de este artículo de investigación se centra en exponer de manera resumida las conceptualizaciones elementales que la literatura científica más reciente que se encuentra disponible respecto a la dermatitis atópica o eccema atópico en niños, para ello, se estima referenciar definiciones, conceptos, de cómo es concebida esta patología, cuáles son sus causas, características, síntomas, comportamiento infantil, tratamiento farmacológico y cuidados preventivos en el hogar.

\section{Materiales y Métodos.}

El desarrollo de este artículo se ha llevado a cabo en el marco de una investigación científica de tipo documental, en la que se ha dispuesto básicamente de computadores personales como herramientas y materiales de trabajo con los que se ejecuta parte de la metodología inherente a la naturaleza investigativa previamente definida por el equipo investigador.

Este desarrollo investigativo se ha limitado a la búsqueda de material bibliográfico por medio del uso de diversas bases de datos consultadas, tales como: MedlinePlus, PubMed, Portal Regional de la BVS, SciELO y ELSEVIER.

En dichas fuentes se efectuó una consulta/búsqueda aleatoria y consecutiva usando las expresiones: dermatitis atópica infantil; dermatitis atópica pediátrica; dermatitis atópica en niños; eccema infantil y eccema en niños; de las que se fueron seleccionando los títulos y materiales bibliográficos concebidos como artículos científicos de revistas especializadas, libros, boletines, 


\section{Dermatitis atópica (ECCEMA) infantil}

Vol. 3, núm. 3., (2019)

Diana Stefania Evangelista Barragan; Gabriela Estefania Jalon Flores; Jorge Efraín Camacho Desidero; Johamelly Maritza Peña Martillo

folletos, tesis de grado, posgrado y doctorado, noticias científicas, entre otros tipos de documentos de interés científico, que fundamentalmente guardaron mayor relevancia con las expresiones antes mencionadas, y además respondieran a los criterios de vigencia y transcripción en los idiomas español e inglés, hasta alcanzar un aproximado de 35 (treinta y cinco) trabajos de los cuales se fueron extrayendo las ideas y planteamientos aquí plasmados.

\section{Resultados.}

"La dermatitis atópica es una condición que se presenta con bastante frecuencia en las mejillas de los niños y que consiste en placas descamativas y rojas diseminadas de manera difusa a través de todo el cuerpo y el rostro" (Instituto National de Salud de USA / NIH, 2018)

El eccema es una afección de la piel en la cual se presentan zonas secas, descamativas, rojas (o de un color más oscuro al del color normal de la piel) y que producen picazón. Cuando continúa por largo tiempo, estas zonas se vuelven gruesas. Esta afección a menudo se asocia con el asma y con las alergias, aunque con frecuencia puede ocurrir sin que se presente ninguna de estas afecciones. El eccema a menudo es hereditario. (NIH, 2017)

Para Garrote \& Bonet (2017):

Clínicamente también se ha observado que parece existir una cierta correlación entre los casos de eccema atópico y los de determinadas patologías como el asma, la fiebre del heno y la rinitis alérgica; es por ello que esta dermatosis está considerada como parte integrante del complejo sintomático denominado "atopia" que afecta el sistema inmunitario. Su desarrollo puede estar influenciado por múltiples factores, sin embargo, los condicionantes genéticos 


\section{Dermatitis atópica (ECCEMA) infantil}

Vol. 3, núm. 3., (2019)

Diana Stefania Evangelista Barragan; Gabriela Estefania Jalon Flores; Jorge Efraín Camacho Desidero; Johamelly Maritza Peña Martillo

ocupan un papel preponderante, estableciéndose que la probabilidad de padecer dermatitis atópica se incrementa en un 50-70\% si existen antecedentes familiares (si uno de los progenitores ha padecido también dicha enfermedad).

De la misma manera, los tratadistas aseguran que lo que aumenta su prevalencia son los cambios en el modo de vida, el aumento de la higiene en la sociedad y los factores externos (polen, ácaros, polvo, contaminación, determinados tensioactivos, metales, entre otros), mismos que, además de poder condicionar el surgimiento de la enfermedad, igualmente pueden influir en el agravamiento de la patología en razón de que, "las funciones inmunitarias del paciente están modificadas y son más sensibles a la acción de determinados elementos que puedan actuar como alérgenos"; inclusive algunos alimentos tales como huevos, leche de vaca, cacahuetes, plátano, pescado, y otros, llegan a ser un factor determinante de entre el 10 a $20 \%$ de los casos. Agregan que en los últimos 10 años sobre esta entidad dermatológica se ha estimado que a alcanzado triplicar el número de casos, probablemente en razón de una mayor concentración de sustancias irritantes en el ambiente.

Aparte exponen que, respecto al modo de vida de las personas que la padecen este tipo de afección, ha sido a través de experimentos que se ha demostrado que las situaciones estresantes o el desequilibrio emocional igualmente propician el desencadenamiento de un nuevo brote de dermatitis atópica, o también, pudieran empeorar notablemente uno ya existente, es por ello que esta dermatitis, a veces, se le reconozca como neurodermatitis. 


\section{Dermatitis atópica (ECCEMA) infantil}

Vol. 3, núm. 3., (2019)

Diana Stefania Evangelista Barragan; Gabriela Estefania Jalon Flores; Jorge Efraín Camacho Desidero; Johamelly Maritza Peña Martillo

Continúan explicando que el exceso de sudor, el sol, el frío, ambientes demasiado calientes y secos, los cambios repentinos de temperatura, bañarse sin aplicar a continuación un agente hidratante adecuado, entre otros factores de tipo físico, también influyen en los síntomas de la dermatitis atópica.

El individuo atópico, si bien es un paciente que no tiene comprometida seriamente su salud orgánica, sufre una merma importante en su calidad de vida por el tipo de sintomatología que lo afecta. En muchos pacientes se manifiestan secundariamente problemas de insomnio, ansiedad, agitación, que condicionan tanto la vida del paciente como la de su entorno. Dato significativamente relevante al tener en cuenta que un alto porcentaje de afectados son niños. (Garrote \& Bonet, 2017)

\section{Causas}

Especialistas concuerdan con que el eccema se relaciona con una variación genética que afecta la capacidad de la piel de proporcionar la protección contra las bacterias, los irritantes y los alérgenos que una normalmente proporciona una piel sana, siendo entonces ésta la razón por la que la piel se vea afectada por factores ambientales, irritantes y alérgenos. (Mayo Clinic, 2018)

En el mismo sentido, en una publicación especial se ha extraído la idea que entes como el Instituto Nacional de Artritis y Enfermedades Musculoesqueléticas y de la Piel (National Institute of Arthritis and Musculoskeletal and Skin Diseases / NIAMS, por sus siglas en inglés), parte de los Institutos Nacionales de Salud de los Estados Unidos de América (NIH, por sus siglas en inglés), y la Academia Americana de Dermatología (American Academy of 


\section{Dermatitis atópica (ECCEMA) infantil}

Vol. 3, núm. 3., (2019)

Diana Stefania Evangelista Barragan; Gabriela Estefania Jalon Flores; Jorge Efraín Camacho Desidero; Johamelly Maritza Peña Martillo

Dermathology / AAD; por sus siglas en inglés) tienen sobre la etiología de este enfermedad. En dicho material se afirma que:

Existen varias hipótesis para explicar las causas de la aparición de DA. Se consideran aspectos genéticos, ya que en un 50 a $70 \%$ de los afectados con DA existen antecedentes de atopia personal o familiar. Ya se han encontrado varios genes relacionados con enfermedades alérgicas siendo de interés para la dermatitis atópica la mutación del gen filaggrin. Esto, sin embargo, no explica el aumento de los casos de DA en las últimas décadas, por lo que se postulan aspectos ambientales, infecciosos, neurovegetativos, alimentarios, entre otros. A su vez, la patofisiología de la DA considera aspectos inmunológicos y de la barrera cutánea. (NIAMS \& AAD, 2018)

\section{Características}

En el lactante (entre los 2 meses y los 2 años)

Afecta aproximadamente del 6 al 15\% de los niños de este grupo y a un tanto menos del $30 \%$ de los lactantes nacidos de padres que padecen alguna enfermedad de tipo alérgico.

Los síntomas regularmente llegan a iniciar entre el cuarto y el sexto mes de vida , presentandose en forma de una dermatosis eritematosa, prurítica y exudativa delimitada a las zonas de "la cara (mejillas, frente, detrás de las orejas), cuero cabelludo, tronco (pecho, espalda y nalgas) y superficies laterales externas de piernas y brazos y parte interna de las muñecas y dedos de las manos (el pulgar es una zona característica)", sin embargo, es posible que igualmente se presente en otros partes del cuerpo. "Las placas eritematoedematosas iniciales evolucionan a 


\section{Dermatitis atópica (ECCEMA) infantil}

Vol. 3, núm. 3., (2019)

Diana Stefania Evangelista Barragan; Gabriela Estefania Jalon Flores; Jorge Efraín Camacho Desidero; Johamelly Maritza Peña Martillo

lesiones papulovesiculosas, con exudación y formación de costras." (Garrote \& Bonet; 2017; p. 30)

En el niño (2-12 años)

Es probable que en el $80 \%$ de los casos de niños con dermatitis atópica desarrollen los primeros síntomas de esta afección antes de cumplir el primer año de edad, sin embargo, casi la mitad de esos mismos casos quedarán exentos naturalmente de las lesiones al cumplir los 2 años de edad, afortunadamente. Cabe destacar que, es probable que disminuya la probabilidad de la remisión temprana de esta afección si su primera aparición se da en un niño mayor de un 1 año de edad.

Las lesiones en niños en edad escolar suelen concentrarse en los pliegues de la piel de las superficies de flexión: cuello, codos, muñecas, rodillas y tobillos. Las lesiones, eccemas secos y descamativos, provocan un intenso picor, y como respuesta al mismo el niño se rasca, lo que facilita la aparición de escoriaciones y fisuras, con el consiguiente agravamiento de las lesiones y aumento del riesgo de sobreinfección. Adicionalmente, en esta etapa pueden aparecer conjuntamente otras manifestaciones cutáneas tales como: queilitis descamativa, piritiasis alba, dermatitis plantar juvenil, piel seca y agrietada en dorso de las manos y pies. (Garrote \& Bonet; 2017; p. 30)

Síntomas

Considerando que esta afección es asumida como un problema crónico de la piel, es notorio que sus síntomas son diferentes en cada niño, pudiendo aparecer en cualquier parte del 


\section{Dermatitis atópica (ECCEMA) infantil}

Vol. 3, núm. 3., (2019)

Diana Stefania Evangelista Barragan; Gabriela Estefania Jalon Flores; Jorge Efraín Camacho Desidero; Johamelly Maritza Peña Martillo

cuerpo o sólo en algunas partes, y tienen la particularidad de desaparecer tan circunstancialmente como inicialmente aparecieron. Existen periodos en que dichos síntomas empeoran (conocidos como: exacerbaciones), y éstos comunes pudieran tratarse de: piel seca, enrojecida y con picazón, y sarpullidos, pudiendo ser éstos tanto secos como supurantes.

Los sarpullidos, comúnmente en los bebes, salen en la cara y en el cuero cabelludo, mientras que, en niños más pequeños, les aparece en los dobleces de los codos y de las rodillas. (AAD, 2016)

\section{Comportamiento infantil}

De acuerdo al trabajo de Yang, Beck, Sekhon, Bhutani, \& Koo (2019) se conoce que existen modestos "estudios de cohortes han demostrado que los niños que sufren de dermatitis atópica son más dependientes y apegados, temerosos y tienen más problemas de comportamiento que los niños no afectados, rasgos que se amplifican en pacientes con enfermedades más graves.”. Además, explican que, particularmente con esta condición, se asocia la alteración del sueño, y al respecto se ha demostrado el aumento de la somnolencia diurna, la irritabilidad y los problemas de disciplina en los pacientes afectados, y así mismo, los niños se encuentran a "un mayor riesgo de afecciones de salud mental, como el trastorno por déficit de atención con hiperactividad, depresión, ansiedad, trastorno de conducta y autismo, lo que puede aumentar enormemente la dificultad de cuidar a estos niños." (Yang, Beck, Sekhon, Bhutani, \& Koo; 2019; p. 67) 


\section{Dermatitis atópica (ECCEMA) infantil}

Vol. 3, núm. 3., (2019)

Diana Stefania Evangelista Barragan; Gabriela Estefania Jalon Flores; Jorge Efraín Camacho

Desidero; Johamelly Maritza Peña Martillo

\section{Tratamientos farmacológicos}

\section{Con prescripción facultativa}

Esteroides tópicos; es uno de los medicamentos indicados desde hace muchos años, los utilizados más ampliamente para este tipo de afección por ser eficaz y seguro cuando es usado de acuerdo con las indicaciones médicas, debido a que es el que sirve para reducir la inflamación (enrojecimiento e hinchazón) y detener la comezón (picazón). Entre sus efectos secundarios menos comunes se citan: el adelgazamiento de la piel, estrías y acné.

Inmunomoduladores tópicos (medicamentos libres de esteroides); medicamento un tanto más reciente que, igualmente, es de los indicados para detener la comezón y reducir la inflamación, pero que se diferencia porque ha demostrado que funciona en un $80 \%$ de los estudios realizados en niños mayores de 2 años.

Los antihistamínicos orales; la eficacia de este tipo de medicamento es mayor en ciertos niños que en otros, y por lo general se puede conseguir varios tipos de estos.

Antibióticos orales; prescritos si se evidencia algún tipo de infección secundaria.

Esteroides orales; regularmente no recomendados en los niños pequeños porque plantean muchos riesgos para la salud, aparte de que, es muy frecuente que la afectada sufra de erupciones de rebote después de que los dejan de tomar. 


\section{Dermatitis atópica (ECCEMA) infantil}

Vol. 3, núm. 3., (2019)

Diana Stefania Evangelista Barragan; Gabriela Estefania Jalon Flores; Jorge Efraín Camacho Desidero; Johamelly Maritza Peña Martillo

Sin prescripción facultativa

Esteroides tópicos (cremas con hidrocortisona o pomadas); funcionan mejor para el eccema leve, proporcionando alivio de la picazón de la piel y disminuyendo la inflamación.

Medicamentos tópicos sin esteroides; proporcionan alivio rápido a la piel seca, con picazón, y tiene pocos efectos secundarios, entre ellos, la sensación de ardor que causan algunos de estos medicamentos por su contenido de alcohol.

Antihistamínicos orales; pueden ayudar a aliviar la picazón (particularmente los que hacen sentir soñolientos a algunas personas). (AAD, 2016)

Recientemente se han venido empleando ungüentos con base de crisaborol como nuevos tratamientos tópicos para los casos leves a moderados de dermatitis atópica, mismo que básicamente actúa disminuyendo los factores de inflamación. Así mismo la FDA () ha aprobado otro medicamento conocido como dupilumab inyectable, el cual se trata de "un anticuerpo monoclonal que disminuye la formación de mediadores de inflamación. Es de mayor utilidad y uso en casos moderados a severos de dermatitis atópica" (NIAMS, NIH \& AAD; 2018; p. 27)

\section{Cuidados en el hogar}

A pesar de que muchos de los niños con eccema tendrán la piel sensible en la edad adulta, la mayoría podrán ir percibiendo el alivio de estos síntomas con la edad, por lo tanto, las claves para reducir las erupciones en este tipo de padecimiento serían: 


\section{Dermatitis atópica (ECCEMA) infantil}

Vol. 3, núm. 3., (2019)

Diana Stefania Evangelista Barragan; Gabriela Estefania Jalon Flores; Jorge Efraín Camacho Desidero; Johamelly Maritza Peña Martillo

- Preferiblemente usar vestimenta holgada y de algodón que coadyuven en la absorción de la transpiración corporal.

- Evitar jabones que resequen la piel y/o cualquier otro producto que previamente haya causado irritación (inclusive los alimentos).

- Evitar tomar baños por largos periodos de tiempo, con agua caliente y/o en tinas (bañeras) con productos para baño corporal (por ejemplo, burbujas) que pueden ocasionar resequedad.

- Aplique crema humectante (o cualquier ungüento hidratante) inmediatamente después del baño para evitar la resequedad.

- En los bebes, especialmente, procurar mantener cortas las uñas de sus manos, y en los niños pequeños, considerar la posibilidad de que para dormir en las noches, usen guantes suaves para reducir el rascado en la piel.

- Consultar al médico especialista en caso de que las medidas preventivas no surtan efecto positivo en cuanto al control del eccema o si se observa el inicio de algún tipo de infección en la piel. (NIH, 2017)

\section{Conclusiones.}

La dermatitis atópica es una enfermedad de la piel muy frecuente en los niños que, al no ser atendida oportunamente, fácilmente pudiera evoluciona hasta alcanzar determinado nivel de exacerbación que ineludiblemente devendría tanto en persistentes molestias físicas como en 


\section{Dermatitis atópica (ECCEMA) infantil}

Vol. 3, núm. 3., (2019)

Diana Stefania Evangelista Barragan; Gabriela Estefania Jalon Flores; Jorge Efraín Camacho Desidero; Johamelly Maritza Peña Martillo

importantes efectos traumáticos a nivel psicológico, por ello, las acciones preventivas que el adulto responsable lleve a cabo son las estrategias idóneas para mantener bajo control esta común enfermedad que día a día tiende a aumentar su prevalencia a nivel mundial, sobre todo en países de bajos ingresos de América Latina, todo esto es, independientemente de que se sigan desarrollando alternativas medicamentosas para mejorar los síntomas y la prevención de esta particular enfermedad y de que las estadísticas aún sostengan que un alto porcentaje de quienes la llegan a padecer suelen mejorar con el tiempo.

\section{Bibliografía.}

AAD. (12 de 10 de 2016). healthychildren.org. (A. A. Pediatric, Productor) Recuperado el 25 de 04 de 2019, de healthychildren.org: https://www.healthychildren.org/Spanish/healthissues/conditions/skin/Paginas/eczema.aspx

Garrote, A., \& Bonet, R. (Enero-Febrero de 2017). Edades en la dermatitis atópica. Farmacia Profesional, 31(1), 28-31. Recuperado el 25 de 04 de 2019, de https://www.elsevier.es/es-revista-farmacia-profesional-3-pdf-X0213932417607158

Instituto National de Salud de USA / NIH. (14 de 10 de 2018). Medlineplus. Recuperado el 25 de 04 de 2019, de Medlineplus: https://medlineplus.gov/spanish/ency/esp_imagepages/2390.htm

Mayo Clinic. (21 de 09 de 2018). mayoclinic.org. Recuperado el 25 de 04 de 2019, de mayoclinic.org: https://www.mayoclinic.org/es-es/diseases-conditions/atopic-dermatitiseczema/symptoms-causes/syc-20353273

NIAMS \& AAD. (Febrero/Marzo de 2018). Dermatitis atópica (eczema): Algunos aspectos sobre su desarrollo y tratamiento. Galenus, 68(1), 27-28. Obtenido de http://www.galenusrevista.com/?Dermatitis-atopica

NIAMS. (10 de 07 de 2018). medlineplus.gov. Recuperado el 25 de 04 de 2019, de medlineplus.gov: https://medlineplus.gov/spanish/eczema.html

NIAMS, NIH \& AAD. (Febrero/Marzo de 2018). Dermatitis atópica (eczema): Algunos aspectos sobre su desarrollo y tratamiento. Galenus, 68(1), 27-28. Recuperado el 25 de 04 de 2019, de http://www.galenusrevista.com/?Dermatitis-atopica 


\section{Dermatitis atópica (ECCEMA) infantil}

Vol. 3, núm. 3., (2019)

Diana Stefania Evangelista Barragan; Gabriela Estefania Jalon Flores; Jorge Efraín Camacho Desidero; Johamelly Maritza Peña Martillo

NIH. (2017). Medlineplus, Enciclopedia Médica (Versión Electrónica). (I. N. USA, Editor, M. Plus, Productor, \& Medline Plus) Recuperado el 25 de 04 de 2019, de Medlineplus: https://medlineplus.gov/spanish/ency/article/003259.htm

$$
\text { (9) (1) }(2)
$$

RECONOCIMIENTO-NOCOMERCIAL-COMPARTIRIGUAL

CC BY-NC-SA

ESTA LICENCIA PERMITE A OTROS ENTREMEZCLAR, AJUSTAR Y CONSTRUIR A PARTIR DE SU OBRA CON FINES NO COMERCIALES, SIEMPRE Y CUANDO LE RECONOZCAN LA AUTORÍA Y SUS NUEVAS CREACIONES ESTÉN BAJO UNA LICENCIA CON LOS MISMOS TÉRMINOS. 